\title{
Health in the palm of your hand-part 2: design and application of an educational module for young people on the risks from smartphone abuse and the opportunities of telemedicine and e-Health
}

\author{
Daniele Giansanti, Giovanni Maccioni
}

Centro TISP, Istituto Superiore di Sanità, Roma, Italy

Contributions: (I) Conception and design: All authors; (II) Administrative support: None; (III) Provision of study materials or patients: All authors; (IV) Collection and assembly of data: D Giansanti; (V) Data analysis and interpretation: D Giansanti; (VI) Manuscript writing: All authors; (VII) Final approval of manuscript: All authors.

Correspondence to: Daniele Giansanti. Centro TISP, Istituto Superiore di Sanità, via Regina Elena 299, 00131, Roma, Italy.

Email: daniele.giansanti@iss.it; gianslele@gmail.com.

\begin{abstract}
Background: The evolution of mHealth is strictly related to the evolution of the mobile-technology and in particular of the smartphone. However, it is increasingly becoming clear that excessive use of the smartphone has implications for health in terms of emerging risks and in particular (I) the text neck, (II) the addiction, (III) the use of deceptive Apps in mHealth and today telemedicine and e-Health solutions to face this are strongly needed. What young people need is a package of solutions that allow them to tackle all three of these problems at the same time through dedicated tools and specific material on these topics. However up to now this is lacking. The purpose of the study was the design and application of a teaching package focused in this field and specific for young people, the target population for the emerging risks.

Methods: A teaching package was developed in a school-work alternation program for the secondary schools at the Italian National Institute of Health, according the Italian Law. It was available on the basis of proposals offered publically. It provided a two weeks stage on the topics: the risks from smartphone abuse and Telemedicine and e-Health solutions.

Results: A specific electronic survey in this field was available at the end of the stage. The survey was submitted to 500 young subjects (50\% male; 50\% females; mean age 15.9 y; std-dev 1.2 y) at school and/or in meeting initiatives. The outcome demonstrated: (I) that the knowledge on the postural risk (text neck) was very poor, while the desiderata of Apps for correcting the posture was very high; (II) the feasibility to obtain feedback on the risk of addiction through a cyber-psychology approach; (III) a worrying position in relation to non-medical Apps confused with the medical ones.

Conclusions: The study demonstrated how the young people mBorn can familiarize with some aspects of telemedicine and e-Health and be at the same time a kind of spreader of a correct vision of the ratio between user and smartphone and the knowledge on both the mTech and the related problems.
\end{abstract}

Keywords: Mobile-health; smartphone; text neck; addiction; telemedicine

Received: 17 June 2020; Accepted: 06 December 2020; Published: 20 July 2021.

doi: $10.21037 /$ mhealth-20-129

View this article at: http://dx.doi.org/10.21037/mhealth-20-129 


\section{Introduction}

The global study is arranged into two companion papers structured as commentaries.

(I) The first paper "Part 1: the risks from smartphone abuse and the role of telemedicine and e-Health" here presented addresses the risks from smartphone abuse and tackle the various gaps and/or the role that Telemedicine and e-Health itself has in this field.

(II) The second paper "Health in the palm of your hand. Part 2: Design and application of an educational module for young people on the risks from smartphone abuse and the opportunities of telemedicine and e-Health" deals with the design and application of a new teaching package/module dedicated to young people, the target population, which faces both the abuse risks and the approach to the problem also based on several Telemedicine and e-Health components

\section{The mobile health: the key role of the smartphone}

\section{The new potential of smartphone}

Mobile-health is today playing an important role in the Telemedicine and e-Health applications (1) furthermore the potential effects of mobile health on health system is significant, even if there are some gaps in development and evaluation of the effectiveness of mobile health interventions. The core element is the smartphone, which, with specifically dedicated applications (App)s, or also with the use of the common ones, as for example the messengers with the group functionalities (2), is more and more presenting as a key element in mobile-health. The new potential of smartphones compared to mobile phones combined with the possibility of interacting in a userfriendly way with the device through the virtual keyboard and simple Applications (App)s and with the decreasing of the costs of the communication have increased the time of use of these devices (3). As it is well known, the smartphone allows access to the network for multimedia entertainment, to communicate via chat and social networks, for video calls, for downloading of contents and applications, and finally for reading textual contents such as books, newspapers.

\section{The flip side of the coin}

The flip side of the coin is highlighted by placing attention to the time of use and to the type of physical activity resulting from the interaction between subject and smartphone
$(3,4)$. In particular a growing increase in time dedicated to smartphone in non-voice activities is recorded (5), which has rapidly grown and is around 3 hours a day (US data). An exposition of this entity appears relevant for the risk to exposure. Particular account must be taken of the fact that the multiple features of the smartphone make it particularly attractive to young people; furthermore, it is precisely among young people that the smartphone has become an indispensable tool not only for traditional phonic communications but also to keep social relations in motion through dedicated Apps such as social networks and messengers. Taking into account these two aspects together with the life expectancy of young people and the early use (from early childhood) of this device, it emerges that young people (adolescent and young adults) represent the "critical population" for the new risks that emerge in the abuse of the smartphone. In the companion paper (3) "Part 1: the risks of smartphone abuse and the role of telemedicine and e-Health" we have faced these new risks and identified the contribution that could play the e-health and m-heath in this field.

\section{The new risks from smartphone abuse and the contribution of telemedicine and e-Health in brief}

The emerging risks from smartphone abuse and the role of telemedicine and e-Health have been fully described in the companion paper (3). For the sake of clarity of the reader we report them in brief below.

\section{The text-neck and the approach of telemedicine and e-Health}

The text neck (3-5) is a definition used in recent years to describe stress injuries repeated and neck pain resulting (I) from an incorrect interaction with the devices (6-9) (II) used for an excessive period of time. This definition is today more used than the other of turtle neck (which is used for the same problems) probably because it is more directly related to typing activities (texting) $(10,11)$ in the smartphone, which among the various activities is the one most connected to the syndrome compared to others possible with the smartphone, such as web browsing. The symptoms range from simple headache up to cervical hernia. Experts of the text neck, to relieve stress due to posture, suggest simple changes in lifestyle. Among the many tips, it is suggested to keep the devices in front of the face or at eye level while watching the display and use both hands and the two thumbs to create a more symmetrical and comfortable position for the spine. Experts also recommend to the users to execute stretching 
exercises and other basic physical exercises that help posture. The smartphone is showing potentialities in this field by means of $m$-health solutions. The smartphone thanks to the accelerometer (used as an inclinometer) can indeed return a biofeedback information to the user related to the inclination (biofeedback) of the device. An alignment of the device with respect to the terrestrial vertical pushes the user to take the device to eye level and to assume a correct posture. Instead an inclination with respect to the terrestrial vertical forces the user to bend the neck and assume one incorrect posture by increasing the load on the cervical vertebrae. Several Apps with this function allowing with vibrotactile and/or visual biofeedback and suggesting postural exercises on the bases of the time spent are available in the Virtual stores. These Apps can act as self-therapy and empowerment tools; record data that can be stored for example in a cloud as a diary/log and available for further statistics. Telemedicine and e-Health can contribute here with these Apps and with the acceptance of effectiveness assessment of them (1) and with specific teaching packages (with particular care on young subjects) available also with e-learning solutions.

\section{The addiction risk and the approach of telemedicine and e-Health}

The new potentialities of the smartphone generate psychological problems such as anxiety, depression (12-14) and addiction. According to works recently published some psychological pathologies such as anxiety, depression have as the source the addiction (12). Scholars, with particular attention to the young subjects have proposed tests to investigate the degree of compromising in the addiction such as the smartphone addiction scale short version (SASSV) (15-17) described in the companion paper (3). The contribution of the telemedicine here is well defined and can be based on the design of a test using the electronic survey tools, as for example Forms (Microsoft, USA). Using these cyber-psychology solutions the test can be widely submitted using the same tools familiar to young subjects such as the social networks and the messengers. Telemedicine and e-Health can therefore contribute here with these solutions and stressing training and information (with particular care on young subjects) also with e-learning solutions.

\section{The risk in the use of deceptive Apps in mobile-health and the approach of telemedicine and e-Health}

There are many Apps that, while not medical $(18,19)$, can be confused with these (20). Apps that record parameters, save them in a cloud and make them available to you on request. Consider for example the Apps for fitness. Without going into it very much, it is enough to point out that many consider the metabolic consumption parameter provided by them to be erroneously reliable and build their own everyday life regime on it. Think also of many Apps that promise you the measurement of some parameters (pressure, temperature, glycaemia) simply by placing your fingertip. The role of telemedicine and e-Health (in addition to launch a capillary regulatory action) taking into account that the major users are young people, is to initiate (I) a training and information course on the correct use of these Apps from a young age also using e-learning tools and surveillance projects in the mainframe of their course of study and also through (II) surveillance and monitoring initiatives conducted through dedicated electronic surveys.

\section{The need of a specific solution centered on the youth}

From the review of the literature reported above, it emerges that solutions exist separately for the three problems mentioned. However, there is a strong need for a solution that simultaneously:

(I) Faces the three problems disseminates them using specific materials web based;

(II) Provides technological solutions based on Telemedicine and e-Health for the self-awareness of these problems;

(III) Offers specific training on 2 and 3 on the youth;

(IV) Allow the youth to disseminate the provided solutions to other subjects.

\section{Purpose of the paper}

This paper with the companion paper (3) "Part 1: the risks from smartphone abuse and the role of telemedicine and e-Health" generally faces the new risks from the smartphone abuse and the role of Telemedicine. In particular the companion paper faces this globally identifying the single aspects and the solutions to focus. This study faces the design and supply of a model of training and information strictly related to this field and on young subjects, who represent the critical populations for these new risks. The complete study will therefore disseminate in details:

(I) The design of a teaching package for training on young subjects involving methodological issues;

(II) The application of the package and the analysis of the outcome. 

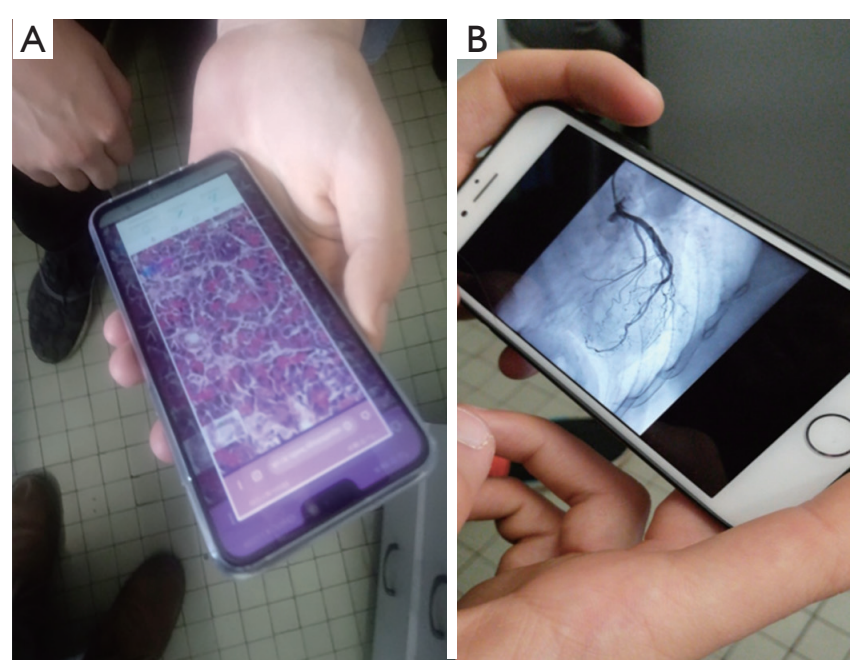

Figure 1 A training phase based on the use of a virtual scope (A) in digital pathology and on a virtual navigation (using WhatsApp to exchange the link pointing a position in a cloud) on hemodynamic traces (B).

\section{Methods}

A specific training package in this field has been designed at the Centro per le Tecnologie Innovative in Sanità Pubblica (Centre TISP) ( https://www.iss.it/?p=76) at the Istituto Superiore di Sanità (ISS) (www.iss.it), a National Body that on the basis of its role has developed a school-work alternation program, the so called Italian Alternanza Scuola e lavoro (ASL) according to the Italian Law 107/2015. The ASL projects (ASL-Ps) are training initiatives for the students of the secondary schools, the ISS, since 2016 (21), activated scientific courses in response to the needs of schools, with more than 24,548 hours offered and the involvement of over 250 researchers.

The recent proposals are available in a dedicated internet address: https://scuolaericerca.iss.it/.

At the end of the course (similar to a stage) the students have the opportunity to apply the experience (I) at their school when they come back interacting with their tutors, teachers and dedicated figures and/or (II) in specific meetings, where researchers encounter the citizens to disseminate and/or involve them in initiatives relevant to the research in the health; one of these initiatives is "La notte europea della ricerca" (22) an initiative contemporary activated in Europe in the public research bodies, usually in September; in Italy it was coordinated by Frascati Scienze, Istituto Nazionale di Fisica Nucleare (https://www.frascatiscienza.it/pagine/notte-europea-dei- ricercatori-2018/.

The specific proposed package (The smartphone a friend but not much-code PS01-) chosen among all packages available at: http://old.iss.it/binary/publ/cont/catalogo_ DEFINITIVO_per_web.pdf provided by the Centre TISP was arranged into the following sub-packages:

(I) A brief summary of the mobile devices evolution and on the inherited risks;

(II) The training on the mobile technology used in mobile-health;

(III) Formation on the new emerging risks: the text neck;

(IV) Formation on the new emerging risks: the addiction risk;

(V) Training on the risk prevention also using the mobile-health Apps focused on the text neck prevention;

(VI) Training on the design of electronic surveys (eS) using a cyber-psychology approach and on the data-analysis for the large investigation of the emerging risks;

(VII) Training on the sector of mobile-health: the non-medical Apps for the fitness and wellness;

(VIII) Training on the deceptive Apps (as for example on those ones that promise to detect your blood pressure or the temperature or even the blood sugar with the simple support of the fingertip).

The package/module comprehends frontal didactics and exercitations.

\section{Results}

All of the students successfully terminated the stage with a high enthusiasm and satisfaction assessed also by means of a properly designed feedback-form. Figure 1 shows a training phase based on the use of virtual scope in digital pathology and on a virtual navigation (using WhatsApp to exchange the link pointing a position in a cloud) (23) on hemodynamic traces (id. 1). Figures 2,3 show a training phase focused on the text neck. After a didactic lesson on the text neck the students are familiarizing with an App for the self-awareness/ therapy/prevention with the text neck (HeadHup-protect your Neck! for Android available in the virtual stores in the WEB) (id.5). This App returns a green visual biofeedback when the smartphone is vertical; this leads the user to raise the device to eye level. Other colored visual biofeedback are used to map a device incorrect angle. Figure 4 shows a training on deceptive Apps: Apps that promise to detect your blood pressure or the 

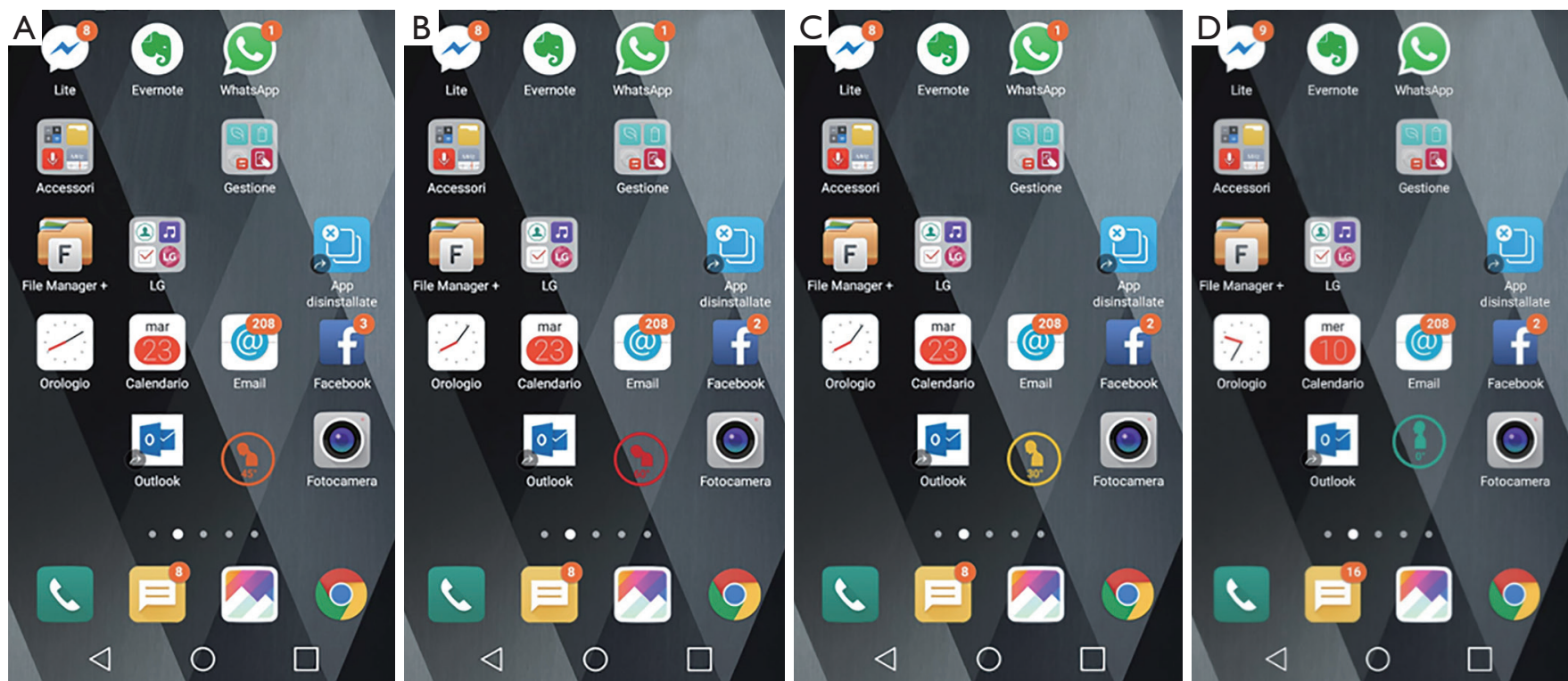

Figure 2 A virtual visual biofeedback indicates the device angle using an icon with different colours in the App focused to the text neck.
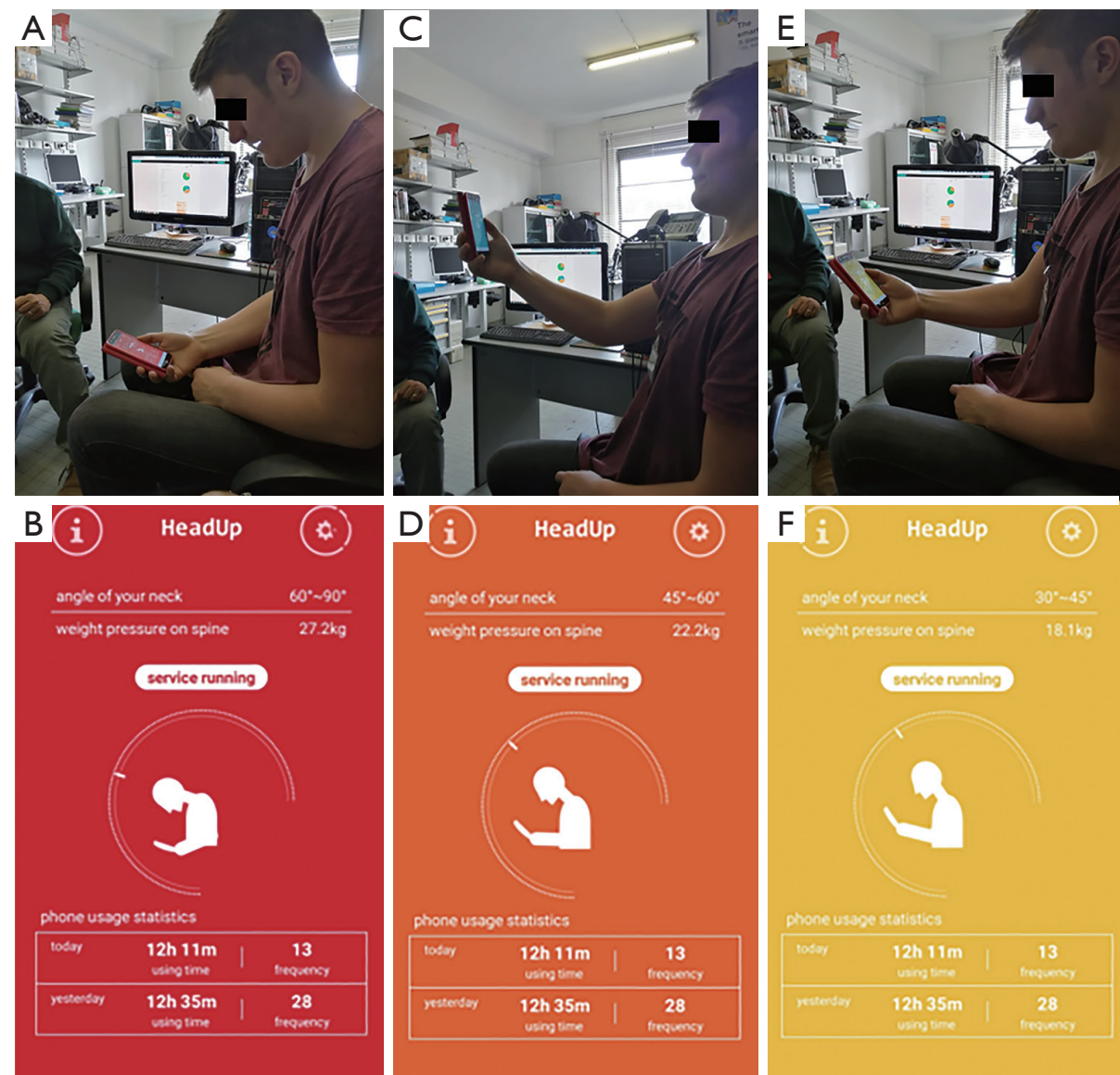

Figure 3 A training phase focused on the text neck. After a didactic lesson on the text neck the students are familiarizing with an App for the self-awareness/therapy and prevention with the text neck. 

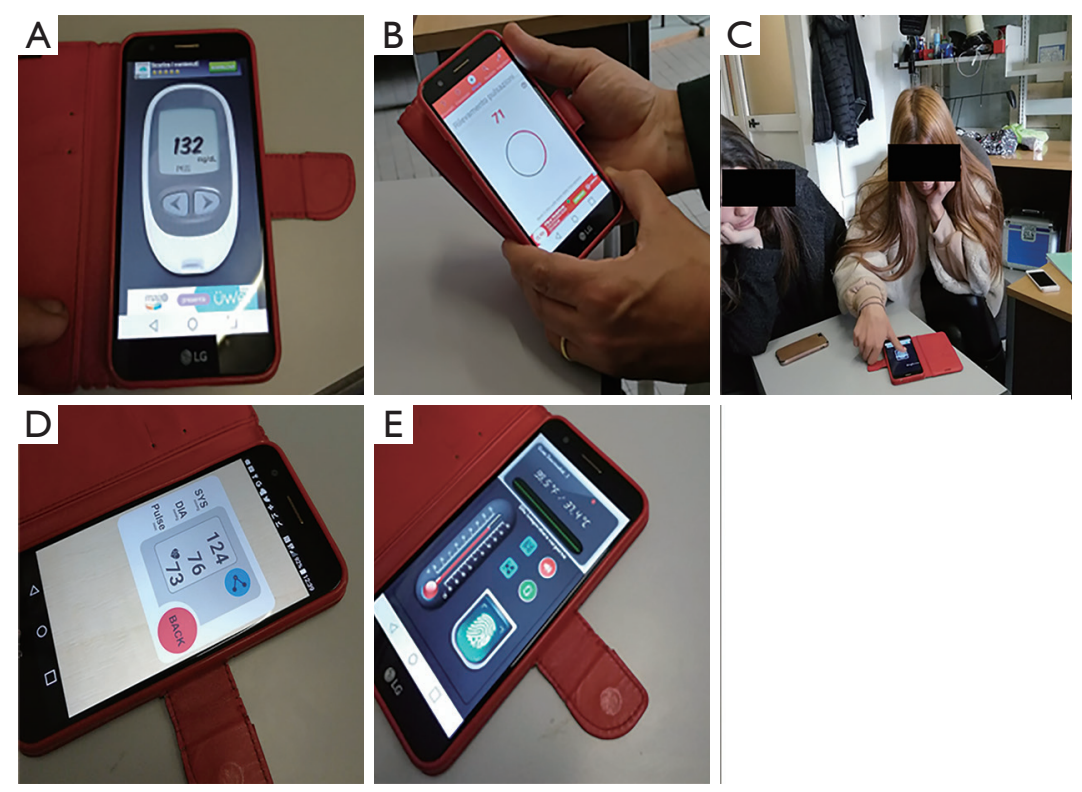

Figure 4 A training phase on deceptive Apps: Apps that promise to detect the blood sugar (A) or your heart-rate (B) or your blood pressure $(\mathrm{C}, \mathrm{D})$ or the temperature (E) with the simple support of the fingertip.

temperature or the heart rate or even the blood sugar with the simple support of the fingertip. Figure 5 highlights the sub packages id-3; id-4; id-5; id-6; id-7; id-8 that integrated together show how telemedicine and e-Health can be of aid to face the new risks from smartphone abuse.

An electronic survey has been proposed as the final product of the stage using Forms (Microsoft, USA). This solution allows the survey delivery based on a cyber-psychology approach using the Apps familiar to young subjects such as the social networks and the messengers. The survey can be enriched using images, designs or emoticon. A report is automatically generated with statistics. After the stage the students had the opportunity to apply the experience (I) wide spreading the product survey at their school when they came back while interacting with their tutors, teachers and dedicated figures and (II) at the meeting "La notte europea della ricerca" above described (22). The final product of this ASL-P comprehends three sections (Figure 6). The first section is a psychologic test on the addiction (3,13-17): the SAS-SV. The second section is on the postural risks. The third section is on the incorrect use of the non-medical Apps. All the questions were based on a vote with six levels $(1=\min ; 6=\max )$. The outcome also represents an exercise in a positioning research action in telemedicine and e-Health. Figure 7 reports the link and the Quick Response Code (QR code) of a section/piece of the electronic survey (eS) relevant to the test SAS-SV (3), extracted and converted into
English for editorial scope with some print screens.

After the data-mining the following considerations are possible: 500 voluntary students ( 250 males; 250 females) participated at the study after agreeing giving an electronic informed consensus. The range of age was $13-19$ years (mean 15.9; std-dev 1.2). Figure 8 returns the outcome from the Test SAS-SV investigating the risk of addiction, this test categorizes the subjects on the basis to the sex and the total score (3) according to three groups (low-risk, high-risk, with addiction). Results indicated that it was present a group of students with a high risk of addiction and that however no students were found addicted. Figures 9-11 show the outcome relevant to the postural risk and opinions on dedicated $m$-health Apps. Each person interviewed in the question asked was able to assign a grade from 1 (minimum) to 6 (maximum). Results indicated that that the knowledge on the postural risk and text neck was very poor (Figures 9,10), while the desiderata of Apps for correcting the posture was very high (Figure 11). The last Figure 12 reports the outcome of the investigation on the deceptive non-medical Apps, elucidating as, in some cases, the mean value of the answers is not so small as desirable.

\section{Discussion and conclusions}

The study has globally faced with the companion paper (3) the new emerging risks from smartphone technology abuse 


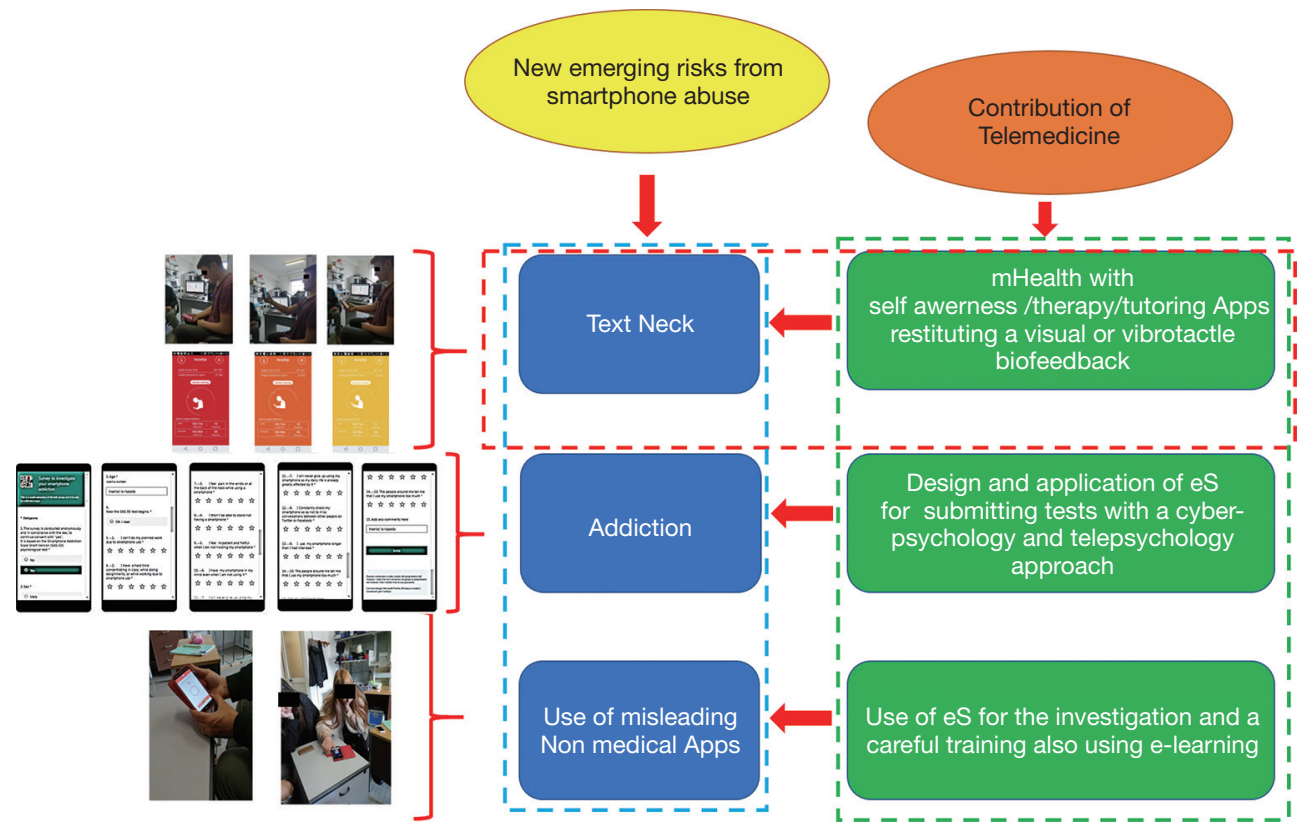

Figure 5 How telemedicine and e-Health can be of aid to face the three new smartphone emerging risks.
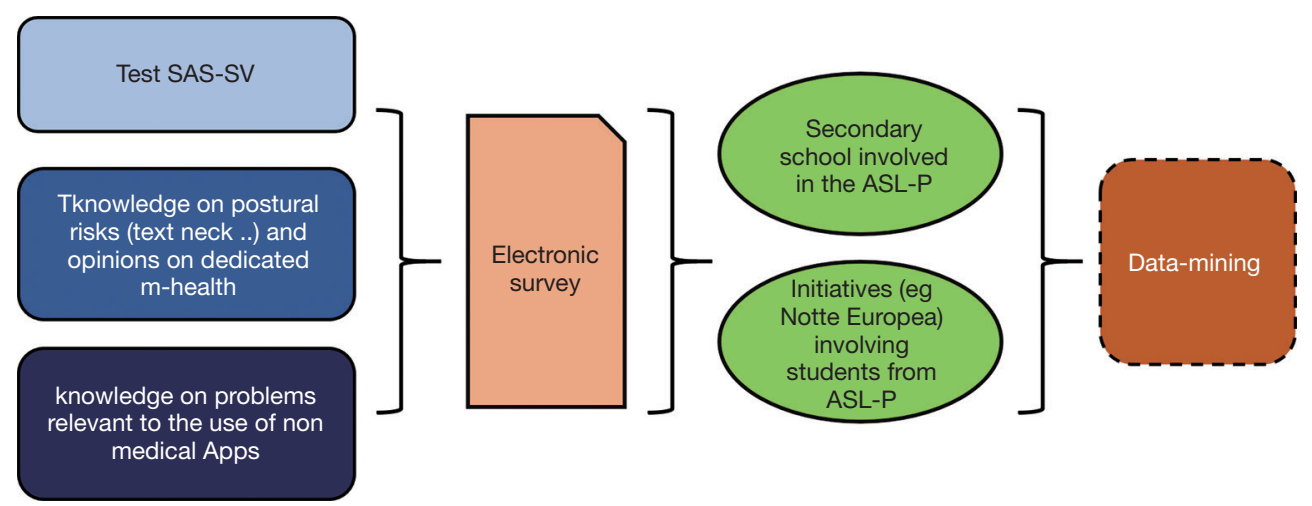

Figure 6 The submission of the Alternanza Scuola e Lavoro Product (ASL-P): from the design up to the data-mining (SAS-SV stands for smartphone addiction scale short version).

and the role of telemedicine and e-Health. The emerging risks were the following:

(I) The text neck a kind of postural neuromuscular risk (4-11);

(II) The addiction risk (12-17);

(III) The risk due to incorrect use of non-medical Apps confoundable with medical (18-20).

The first part of the study in the companion paper (3) focused in details to these risks and to the role of the telemedicine and e-Health in this field.

The global study terminated with this work illustrated in this contribution that reports the design and application of a teaching package/module in this field dedicated to young students of the secondary schools. The package was developed in a school-work alternation program, according the Italian Law 107/2015 and could be chosen on the basis of proposals offered publically. It provided a two weeks stage. At the end of the stage a final product was available consisting on an electronic survey to be used for a specific investigation in this field. After a submission process of the survey, data were available after a datamining. From the data-mining emerged: (I) a poor knowledge of the postural risks and a 

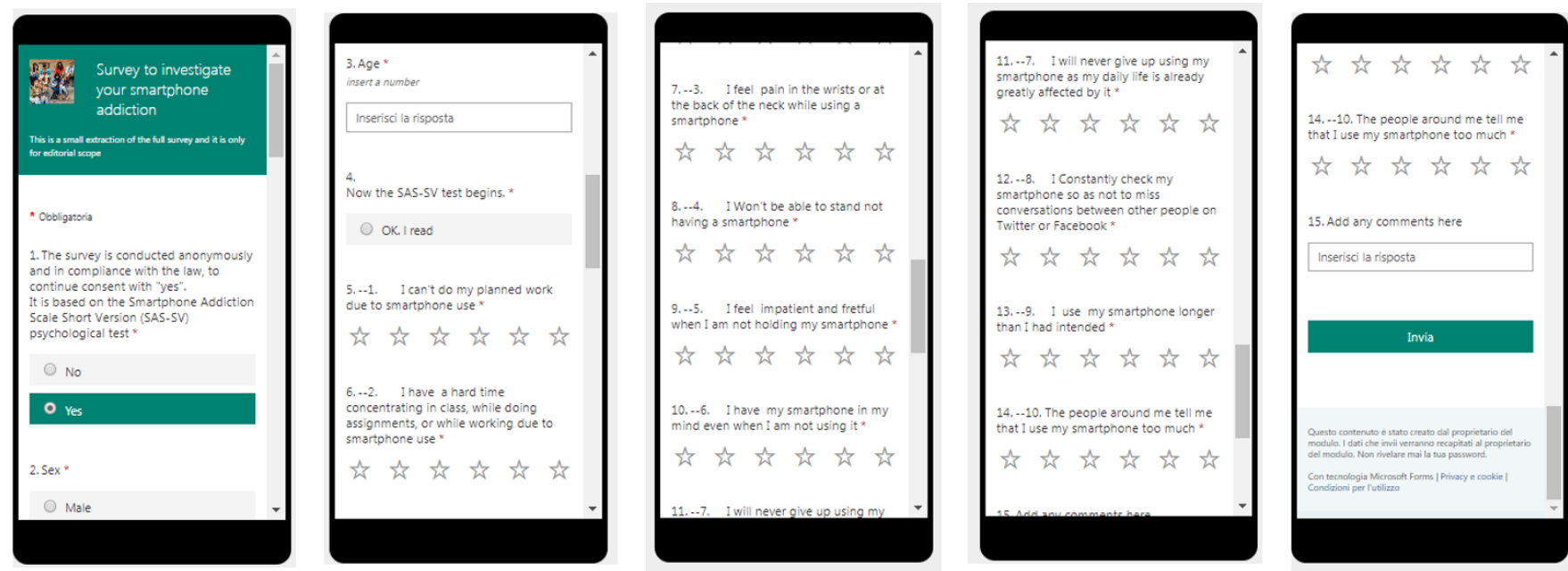

\section{Link of the eS:}

https://forms.office.com/Pages/ResponsePage.aspx?id=DQSIkWdsWOyxEjajBLZtrQAAAAAAAAAAAAZAAOUXdFhURVpWTVhFTDBRUEFOUF YzSIVXWEsyWjJaMC4u

Link of the eS report:

https://forms.office.com/Pages/AnalysisPage.aspx?id=DQSIkWdsWOyxEjajBLZtrQAAAAAAAAAAAAZAAOUXdFhURVpWTVhFTDBRUEFOUFY zSIVXWEsyWjJaMC4u\&AnalyzerToken=iqzeoLQAAHfJC7uGHEYtfJXtXaSdhoPS
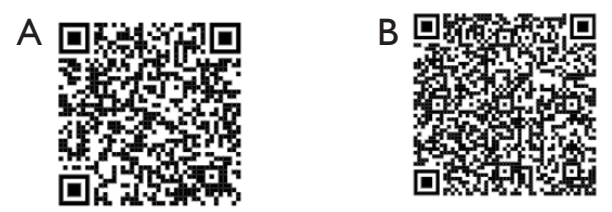

Figure 7 The eS: the print screens; the link internet and (A) Quick Response Code ; the link of the online report automatically updated at each eS-submission with (B) the Quick Response Code.

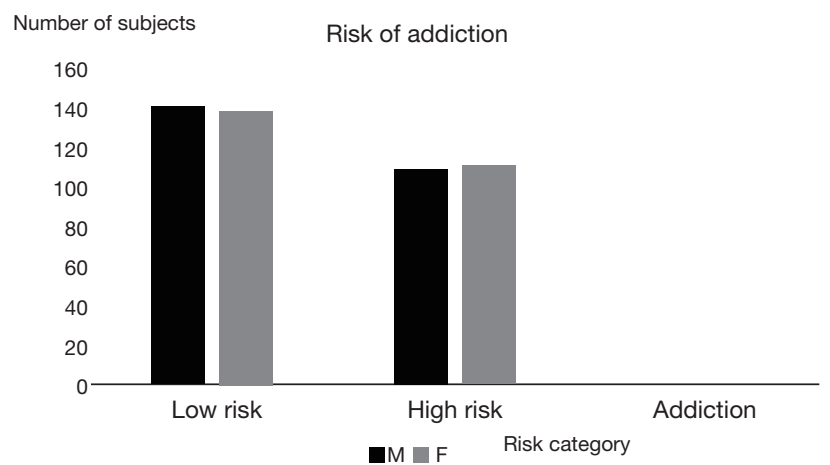

Figure 8 The outcome from SAS-SV test conducted using a cyberpsychology approach. Results are grouped for the level of risks ( $M$ and $\mathrm{F}$ stands for males and females).

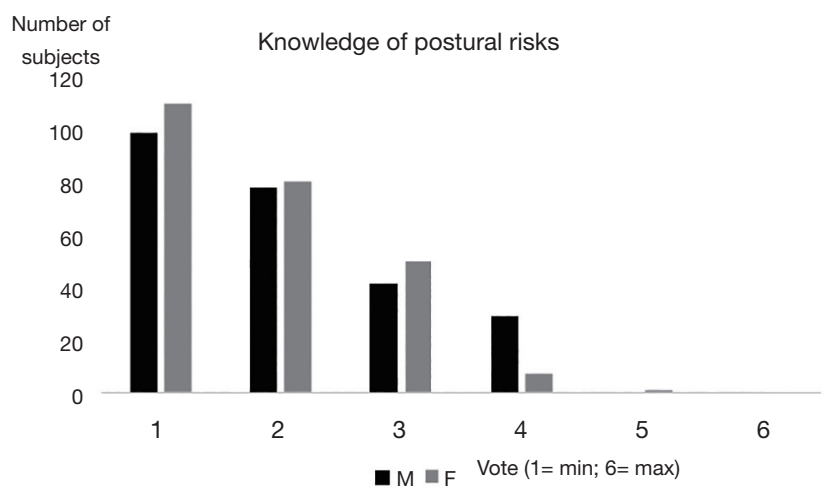

Figure 9 The knowledge on the postural risk. Each person interviewed in the question asked was able to assign a grade from 1 (minimum) to 6 (maximum), in particular " 1 " indicates the lowest knowledge on postural risks, while " 6 " indicates the highest one (M and $\mathrm{F}$ stands for males and females). 


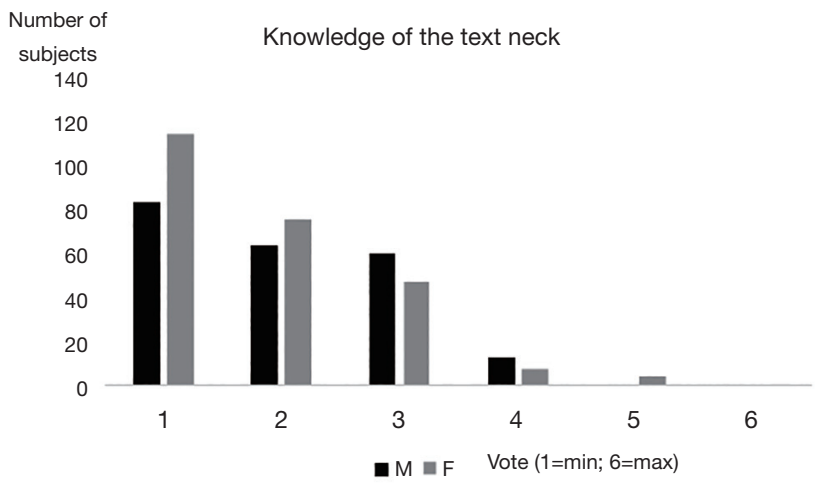

Figure 10 The knowledge on the text neck. Each person interviewed in the question asked was able to assign a grade from 1 (minimum) to 6 (maximum), in particular " 1 " indicates the lowest knowledge on the "text neck", while " 6 " indicates the highest knowledge on the "text neck". ( $M$ and F stands for males and females).

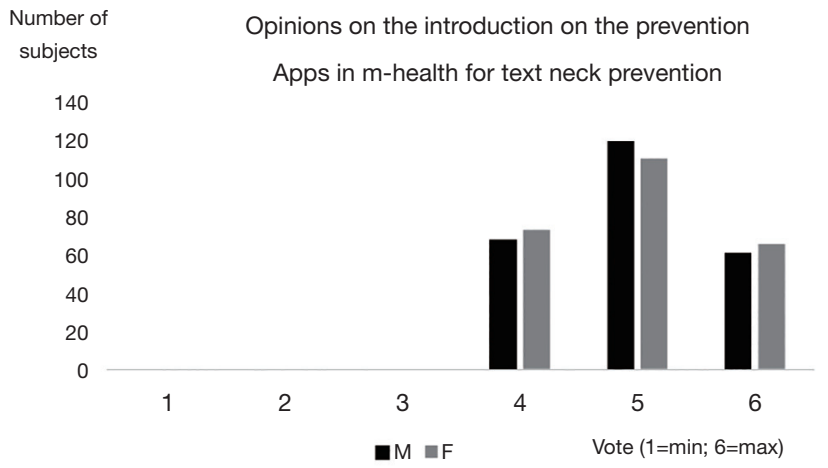

Figure 11 Opinions on the introduction of Apps for the text neck prevention. Each person interviewed in the question asked was able to assign a grade from 1 (minimum) to 6 (maximum), in particular " 1 " indicates the lowest wish on the "introduction on the prevention Apps....", while " 6 " indicates the highest wish on the "introduction on the prevention Apps...". ( $M$ and $F$ stands for males and females).

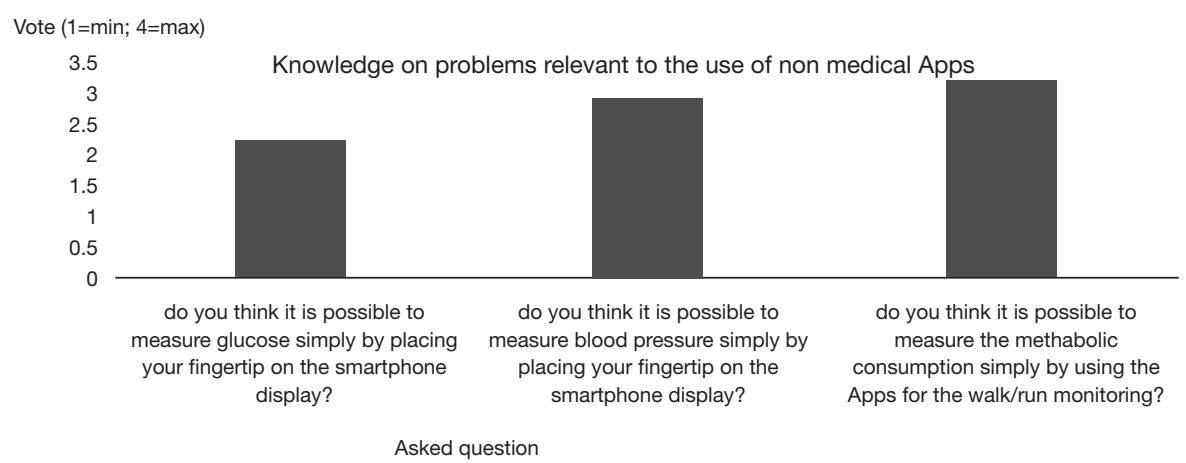

Figure 12 Knowledge on problems relevant to the use of non-medical Apps. Each person interviewed in the question asked was able to assign a grade from 1 (minimum) to 4 (maximum), in particular " 1 " indicates the lowest knowledge on the object proposed in the question, while " 4 " indicates the highest knowledge on the objects proposed in the question.

high desire of prevention Apps in this field; (II) a high risk of addiction for a large group of participants in the study (III) a worrying relationship with the deceptive non-medical Apps.

The overall study considering the two companion papers shows the following added values.

The first added value is an overview to the emerging risks due to smartphone.

The second added value is the model of intervention of the telemedicine and e-Health in this field with the three components of e-health m-health and e-learning.

The third added value is a module/package for a training path in this field dedicated to young subjects (students of the secondary schools).

The last fourth added value is the outcome from the application of this module/package representing a sample of a usefulness intervention on young subjects also demonstrating how the young people mobile-born can familiarize with some aspects of telemedicine and e-Health and be at the same time a kind of diffusor of a correct vision of the ratio between user and smartphone.

\section{Acknowledgments}

Funding: None. 


\section{Footnote}

Conflicts of Interest: Both authors have completed the ICMJE uniform disclosure form (available at http://dx.doi. org/10.21037/mhealth-20-129). DG serves as an unpaid editorial board member of $m$ Health from May 2019 to April 2021. The other authors have no conflicts of interest to declare.

Ethical Statement: The authors are accountable for all aspects of the work in ensuring that questions related to the accuracy or integrity of any part of the work are appropriately investigated and resolved. The ethical approval was not considered necessary, even the consent form was not necessary as the surveys were conducted anonymously.

Open Access Statement: This is an Open Access article distributed in accordance with the Creative Commons Attribution-NonCommercial-NoDerivs 4.0 International License (CC BY-NC-ND 4.0), which permits the noncommercial replication and distribution of the article with the strict proviso that no changes or edits are made and the original work is properly cited (including links to both the formal publication through the relevant DOI and the license). See: https://creativecommons.org/licenses/by-nc-nd/4.0/.

\section{References}

1. de la Torre-Díez I, López-Coronado M, Vaca C, et al. Cost-utility and cost-effectiveness studies of telemedicine, electronic, and mobile health systems in the literature: a systematic review. Telemed J E Health 2015;21:81-5.

2. Williams V, Kovarik C. WhatsApp: An Innovative Tool for Dermatology Care in Limited Resource Settings. Telemed J E Health 2018;24:464-8.

3. Giansanti D, Maccioni G. Health in the palm of your hand-part 1: the risks from smartphone abuse and the role of telemedicine and e-Health. mHealth 2021;7:49.

4. Toh SH, Coenen P, Howie EK, et al. The associations of mobile touch screen device use with musculoskeletal symptoms and exposures: a systematic review. PLoS One 2017;12:e0181220.

5. Neupane S, Ifthikar Ali UT, Mathew A. Text-Neck syndrome- systemic review. Imperial J Interdisciplinary Res 2017;3:141-8.

6. Lee S, Kang H, Shin G. Head flexion angle while using a smartphone. Ergonomics 2015;58:220-6.
7. Hansraj KK. Assessment of stresses in the cervical spine caused by posture and position of the head. Surg Technol Int 2014;25:277-9.

8. Cuéllar JM, Lanman TH. "Text neck": an epidemic of the modern era of cell phones? Spine J 2017;17:901-2.

9. Choi JH, Jung MH, Yoo KT. An analysis of the activity and muscle fatigue of the muscles around the neck under the three most frequent postures while using a smartphone. J Phys Ther Sci 2016;28:1660-4.

10. Gustafsson E, Thomée S, Grimby-Ekman A, et al. Texting on mobile phones and musculoskeletal disorders in young adults: A five-year cohort study. Appl Ergon 2017;58:208-14.

11. Gustafsson E, Johnson PW, Lindegård A, et al. Technique, muscle activity and kinematic differences in young adults texting on mobile phones. (PMID) Ergonomics 2011;54:477-87.

12. Peper E, Harvey R. Digital addiction: increased loneliness, anxiety, and depression. Neuroregolation 2018;5:3-8.

13. De Pasquale C, Sciacca F, Hichy Z. Smartphone addiction and dissociative experience: an investigation in Italian adolescent aged between 14 and 19 years. International Journal of Psychology \& Behavior Analysis 2015;1:109.

14. Elhai JD, Dvorak RD, Levine JC, et al. Problematic smartphone use: conceptual overview and systematic review of relations with anxiety and depression psychopathology. J Affect Disord 2017;207:251-9.

15. Kwon M, Kim DJ, Cho H, et al. The smartphone addiction scale: development and validation of a short version for adolescents. PloS One 2013;8:e83558.

16. Lopez-Fernandez O. Short version of the smartphone addiction scale adapted to Spanish and French: towards a cross-cultural research in problematic mobile phone use. Addict Behav 2017;64:275-80.

17. De Pasquale C, Sciacca F, Hichy Z. Italian validation of smartphone addiction scale short version for adolescents and young adults (SAS-SV). Psichology 2017;8:1513-8.

18. Giansanti D. Introduction of medical Apps in telemedicine and e-health: problems and opportunities. Telemed J E Health 2017;23:773-6.

19. Censi F, Mattei E, Triventi M, et al. Regulatory frameworks for mobile medical applications. Expert Rev Med Devices 2015;12:273-8.

20. Baldani G. Regolamentare l'infinito: La sfida della Food and Drug Administration. Salute e Società 2014:171-5.

21. Salinetti S, De Castro P, Barbaro MC, et al. Alternanza scuola lavoro in ISS. Riflessioni a tre anni di attività (School-work alternation in ISS. Reflections on three years 
of activity, Newsletter Bullettin of the Istituto Superiore di Sanità). Notiziario dell'Istituto Superiore di Sanità 2018;31:3-7.

22. Rossi AM, Barbaro MC, Salinetti S, et al. La notte europea dei ricercatori: un successo in crescita (European researchers' night: a growing success, Newsletter Bullettin

doi: $10.21037 /$ mhealth-20-129

Cite this article as: Giansanti D, Maccioni G. Health in the palm of your hand-part 2: design and application of an educational module for young people on the risks from smartphone abuse and the opportunities of telemedicine and e-Health. mHealth 2021;7:48. of the Istituto Superiore di Sanità). Notiziario dell'Istituto Superiore di Sanità 2018;31:13-6.

23. Giansanti D, Pochini M, Giovagnoli MR. How tablet technology is going to change cooperative diagnosis in the cytology e-laboratory. Telemed J E Health 2013;19:991-3. 